\title{
Adaptive and Assortative Mating Scheme for Evolutionary Multi-Objective Algorithms
}

\author{
Khoi Le and Dario Landa-Silva \\ Automated Scheduling, Optimisation and Planning Research Group \\ School of Computer Science, The University of Nottingham \\ $\{\mathrm{kxl}, \mathrm{jds}\} @ \mathrm{cs}$.nott.ac.uk
}

\begin{abstract}
We are interested in the role of restricted mating schemes in the context of evolutionary multi-objective algorithms. In this paper, we propose an adaptive assortative mating scheme that uses similarity in the decision space (genotypic assortative mating) and adapts the mating pressure as the search progresses. We show that this mechanism improves the performance of the simple evolutionary algorithm for multi-objective optimisation (SEAMO2) on the multiple knapsack problem.
\end{abstract}

\section{Introduction}

Selection plays an important role within evolutionary algorithms in selecting individuals for survival and selecting parents for recombination. Here, we are interested in mating schemes, i.e. the selection of parents for recombination within evolutionary multi-objective (EMO) algorithms. A number of mating schemes have been proposed in the literature including: fitness proportionate selection, tournament selection, rank-based selection, ancestry selection and assortative mating among others. In fitness proportionate selection, parents are chosen based on a probability proportional to their fitness compared to the rest of the population. In tournament selection, a group of individuals (usually two) is chosen (usually uniformly) from the population and the fittest individual from this group is selected as parent. In rank-based selection, individuals are first sorted according to some criteria (usually fitness) and a mapping function is used to assign a selection probability to each individual according to its rank in the ordering. In ancestry selection individuals are organised in clans and parents are usually selected from different clans. In assortative mating (inspired on natural genetics), individuals are selected based on their similarity (in the objective or the decision space) based on the assumption that recombining parents that 'look' alike produces better offspring. Some mating schemes incorporate some form of restricted mating (proposed by Goldberg [6] where recombination is allowed only if parents meet certain criteria. For reviews on mating schemes and their performance of single-objective evolutionary algorithms see [1]

Despite the various restricted mating schemes that have been investigated for single-objective evolutionary algorithms, the emphasis within EMO algorithms has been mainly on mechanisms to select individuals for survival. In Paretobased multi-objective optimisation the goal is to find a set of non-dominated

N. Monmarché et al. (Eds.): EA 2007, LNCS 4926, pp. 172 183, 2008.

(C) Springer-Verlag Berlin Heidelberg 2008 
solutions that is as close as possible to the Pareto optimal front and also well spread and distributed over the trade-off surface [24]. Therefore, most modern EMO algorithms incorporate selection mechanisms, like density-based selection and rank-based selection, in combination with elistism and archiving strategies to ensure the survival of good non-dominated solutions [2,414. Also, most EMO algorithms use tournament or other basic selection mechanism for choosing parents and in most cases selection is based on fitness. Some restricted mating schemes have been investigated in the context of EMO algorithms but to a lesser extent than for single-objective evolutionary algorithms. In their book, Coello Coello et al. ([2, p. 201) express that restricted mating has not been fully investigated for EMO algorithms. They also note that there is no conclusive evidence to support whether restricted mating is beneficial or detrimental for the performance of these algorithms. Coello Coello et al. also suggest that experiments investigating the issue of restricted mating should benefit the literature on EMO algorithms. In this paper, we propose an adaptive assortative mating scheme 3] for evolutionary multi-objective optimisation. That is, parents are chosen based in their similarity in the decision space and the similarity threshold or mating pressure $\sigma_{\text {mating }}$ is adapted during the search. In Section 2 we give a more detailed account of related work. In Section 3 we describe our proposed mating scheme and how this is incorporated into SEAMO2 (simple evolutionary algorithm for multi-objective optimisation) [15]. Section 3 also describes the experimental setting and our results. Final remarks are given in Section 4.

\section{Mating Schemes for EMO Algorithms}

We refer to the $k$-optimisation problem in which the aim is to optimise the function $f(x)=\left(f_{1}(x), f_{2}(x), \ldots, f_{k}(x)\right)$ subject to $x \in X$, where $x$ represents the decision vector, $X$ represents the set of all feasible solutions, $f(x)$ represents the objective vector and each $f_{i}(x)$ represents the value of the $i$-th objective. Within a set $\mathbf{S}$ of solutions, solution $x$ is said to be non-dominated if there is no solution in $\mathbf{S}$ that is better than $x$ in each of the $k$ objectives. Then, $x$ is said to be Pareto-optimal if $x$ is non-dominated with respect to the set $X$.

Now we briefly review restricted mating schemes that have been implemented into EMO algorithms. We focus on schemes proposed recently and that restrict mating based on similarity, i.e. assortative mating. For an overview of previous mating schemes within EMO algorithms refer to the book by Coello Coello et al. ([2], p. 201). Restricted mating has been usually implemented using the $\sigma_{\text {mating }}$ parameter which defines a mating radius or similarity threshold, which can be perceived as the mating pressure. Individuals are not allowed to mate if the distance between them (objective or decision space) is larger than $\sigma_{\text {mating }}$. Kim et al. [13] incorporated neighbourhood crossover into SPEA2 to rank individuals according to how close they are in the objective space and used binary tournaments to select parents. Few years ago, Ishibuchi and Shibata started an investigation into the effect of restricted mating on the performance of the 
well-known NSGA2 and SPEA algorithms. In 2003 they proposed a restricted mating scheme based on the similarity between parents (assortative mating) 9]. Later, they modified their approach by incorporating a second layer to select parent A 10. Their restricted mating scheme works as follows:

1. A set $S_{A}$ of $\alpha$ candidates is chosen using iterative binary tournaments.

2. The center vector (average solution) $\bar{f}(x)=\left(\bar{f}_{1}(x), \bar{f}_{2}(x) \ldots \bar{f}_{k}(x)\right)$ in the objective space in $S_{A}$ is calculated where $\bar{f}_{i}(x)=1 / \alpha \sum_{j=1}^{\alpha} f_{i}\left(x_{j}\right)$ for $i=$ $1,2, \ldots, k$.

3. The solution in $S_{A}$ that is most dissimilar (in the objective space) to $\bar{f}(x)$ is chosen as parent $\mathrm{A}$.

4. A set $S_{B}$ of $\beta$ candidates is chosen using iterative binary tournaments.

5. The solution in $S_{B}$ that is most similar to parent A (in the objective space) is chosen as parent B.

In 10. Ishibuchi and Shibata observed that their modified mechanism was capable of improving both convergence and diversity in SPEA and NSGA2. However, they also noted that the parameters $\alpha$ and $\beta$ needed to be carefully adjusted to strike the balance between diversity and convergence speed. Note also that in [10] Ishibuchi and Shibata used similarity in the objective space only. In 2004, they reported further experiments to investigate the effect of the mating pressure parameters $(\alpha$ and $\beta$ ) and also the effect of similarity (in the objective space) when selecting parents A and B [1]. They tried their restricted mating mechanism in a number of operation modes resulting from combining different settings: $\alpha=\{1,2,3, \ldots\} ; \beta=\{1,2,3, \ldots\}$; parent A being similar or dissimilar to $\bar{f}(x)$; parent $\mathrm{B}$ being similar or dissimilar to parent A. Once again, they observed that convergence speed and diversity were affected by the settings of $\alpha$ and $\beta$. They also expressed that there is a need to set $\alpha$ and $\beta$ automatically in their mating scheme. More recently, Ishibuchi and Shibata reported yet more experiments in which they observed that recombining similar parents (which is controlled by varying $\beta$ ) had a positive impact on the performance of NSGA2, although they also observed that recombination seems to be less important than mutation on that particular algorithm [12. In [12 they considered similarity in the objective and the decision space but only when selecting parent $\mathrm{B}$ and observed no significant difference in their results.

In summary, the investigations by Ishibuchi and Shibata have considered fitness-based binary tournaments and distance in the objective space to choose parent A. For selecting parent B, they employed fitness-based binary tournaments and distance both in the objective space and the decision space. The mating pressure is controlled by the number of tournaments $(\alpha$ and $\beta$ ) and by the target similarity to select parent $\mathrm{A}$ (with respect to the center vector $\bar{f}(x)$ ) and parent B (with respect to parent A). Their results have shown that although their mating scheme is able to improve the performance of SPEA and NSGA2, careful adjustment of the parameters is required to strike the balance between convergence and diversity according to the problem size. 


\section{The Adaptive Assortative Mating Scheme}

\subsection{The Experimental Setting}

We propose an adaptive assortative mating scheme for selecting parents in EMO algorithms. The proposed mating scheme does not use tournaments, it uses similarity in the decision space and changes the mating pressure $\sigma_{\text {mating }}$ as the search progresses. Therefore, this scheme differs from those proposed by Kim et al. 13 and Ishibuchi and Shibata 11. In the proposed dynamic assortative mating scheme two individuals are considered for reproduction only if their dissimilarity (difference between their gene structures) is above a threshold $\sigma_{\text {mating }}$. For the experiments in this paper, we incorporate the proposed assortative mating scheme into the SEAMO2 algorithm [15] and carry out experiments on the multiple knapsack problem. We chose SEAMO2 because it is a simple evolutionary algorithm for multi-objective optimisation that relies mainly on its replacement strategy and it was shown to outperform more elaborate EMO algorithms like NSGA2 and SPEA2 on the multiple knapsack problem [15. In this paper, with refer as SEAMO2(RM) to the SEAMO2 approach using the proposed scheme for restricted mating. Then, we focus our experiments in comparing the performance of SEAMO2(RM) against SEAMO2 [15], SPEA2 [16], NSGA2 [5] and SEAMO2(I) (the SEAMO2 algorithm using Ishibuchi and Shibata's mating strategy [10]) on the multiple knapsack problem. We use the instances with two, three and four knapsacks (with population size of 250, 300 and 350 respectively) and 750 items proposed in [17]. We carry out short, medium and long runs, 500, 960 and 1920 generations respectively, to investigate the performance of SEAMO2(RM). Results from 30 independent runs for each experiment are used for statistical analysis and discussion. We use two metrics, the size of the space covered $\mathcal{S}$ and the coverage of two sets $\mathcal{C}$ (see [17 for details on $\mathcal{S}$ and $\mathcal{C}$ ).

\subsection{Similarity Measurement}

In this paper, the dissimilarity or distance in the decision space between solutions to the multiple knapsack problem is measured as follows:

Individual $s=\left\{g_{i} \mid i: 0 . .(n-1)\right\}$

where $n$ is the number of genes in the individual representation

$$
g_{i}=\left\{\begin{array}{l}
0: \text { gene } i \text { is not in the gene structure of individual } s \\
1: \text { gene } i \text { is in the gene structure of individual } s
\end{array}\right.
$$

the similarity and dissimilarity between individuals $s_{1}$ and $s_{2}$ are as follows:

$$
\begin{aligned}
\operatorname{simil}\left(s_{1}, s_{2}\right) & =\frac{\left|\left\{i \mid g_{1_{i}}=1 \wedge g_{2 i}=1\right\}\right|}{\left|\left\{i \mid g_{1_{i}}=1 \vee g_{2 i}=1\right\}\right|} \\
\operatorname{diff}\left(s_{1}, s_{2}\right) & =1-\operatorname{simil}\left(s_{1}, s_{2}\right)
\end{aligned}
$$

The recombination of $s_{1}$ and $s_{2}$ is allowed if and only if $\operatorname{diff}\left(s_{1}, s_{2}\right) \geq \sigma_{\text {mating }}$ (where $0 \leq \sigma_{\text {mating }} \leq 1$ ). Setting the value of the mating pressure $\sigma_{\text {mating }}$ is important and is discussed in the following sections. 
Note that the above definition of similarity is only valid for solutions to the multiple knapsack problem as encoded in this paper. If the similarity between two solutions of a problem is measured as a percentage, the proposed mating scheme can still be implemented as described later in this paper. Therefore, the generality of the proposed mating scheme is not affected by the encoding of solutions or the method used to measure similarity.

\subsection{Static Setting of the Mating Pressure}

We first describe a simple strategy to preset $\sigma_{\text {mating }}$ before starting the search and this value remains unchanged throughout the evolutionary process. We first calculate the value of $\operatorname{diff}\left(s_{m}, s_{n}\right)$ for every pair of individuals $s_{m}$ and $s_{n}$ in the population. Then, we calculate the range using the minimum and maximum values, i.e. $\operatorname{diff}($ range $)=\left(\max \left(\operatorname{diff}\left(s_{m}, s_{n}\right)\right)-\min \left(\operatorname{diff}\left(s_{m}, s_{n}\right)\right)\right)$. We preset $\sigma_{\text {mating }}$ to a value in this range. Otherwise, if $\sigma_{\text {mating }}$ is set to a value smaller than $\min \left(\operatorname{diff}\left(s_{m}, s_{n}\right)\right)$ the selection of parents becomes uniform. Also, if $\sigma_{\text {mating }}$ is set to a value greater to $\max \left(\operatorname{diff}\left(s_{m}, s_{n}\right)\right)$ no pair of individuals $\left(s_{m}, s_{n}\right)$ would satisfy the selection condition for recombination.

In order to set $\sigma_{\text {mating }}$ to an appropriate value within diff(range) we could let the population to evolve for a limited number of generations and observe the trend on the values of $\operatorname{diff}\left(s_{m}, s_{n}\right)$ in the whole population. We carried out a simple experiment on the multiple knapsack problem and allowed the population to evolve for 100 generations recording diff(range) in every generation. We observed that diff(range) reduces significantly from $60 \%-70 \%$ in the first few generations to $0 \%-35 \%$ in later generations. Therefore, we set $\sigma_{\text {mating }}$ to a value in the range of $(0.0,0.3)$. Next, we carried out experiments using eleven different values of $\sigma_{\text {mating }}: 0.050,0.075, \ldots, 0.300$ in SEAMO2(RM). Results from 30 independent runs are reported in Figure 1. Note that we only show results for six values of $\sigma_{\text {mating }}$ which are representative of all our experimental data. The box-plots in Figure 1 correspond to the percentage of non-covered objective space, i.e. smaller values indicate better algorithm performance. One box-plot is given for each algorithm: NSGA2, SPEA2, SEAMO2, and SEAMO2(RM) using different values of $\sigma_{\text {mating }}$.

Figure 1 shows clearly that with respect to the size of the space covered $\mathcal{S}$ the proposed mating scheme has a positive effect on the performance of SEAMO2(RM). In general, we can see that the performance of SEAMO2(RM) using a preset value of $\sigma_{\text {mating }}$ is consistent over the 30 independent runs (size of the boxplot). There is a significant improvement by applying a higher mating pressure (i.e. increasing the value of $\sigma_{\text {mating }}$ ). However, we can also observe that there is an upper limit for the mating pressure after which SEAMO2(RM) starts to perform worse. We can see in Figure 1 that this upper limit is about $25 \%$ for the 2-knapsack problem (Figure 1(a) 1(b)], between 25\%-30\% for the 3knapsack problem (Figure 1(c), 1(d) , and slighly above 30\% for the 4-knapsack problem (Figure 1(e), 1(f)). This is simply because when $\sigma_{\text {mating }}$ goes above a given value, no parents can be found that satisfy the restricted mating condition. We omit full results for the $\mathcal{C}$ metric (all experimental results are available 


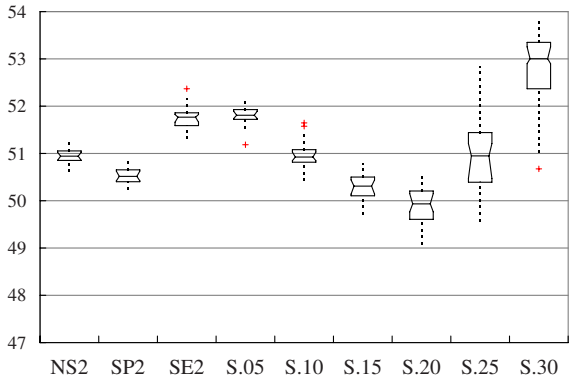

(a) ks2-500

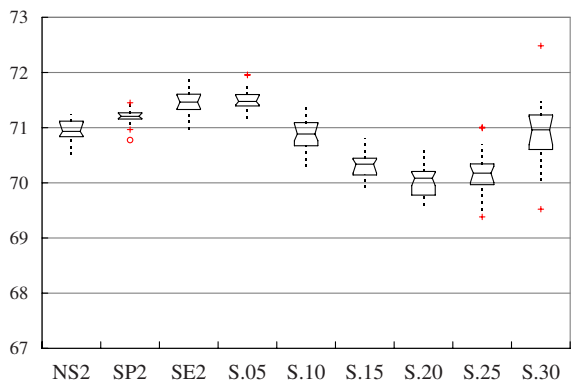

(c) ks3-500

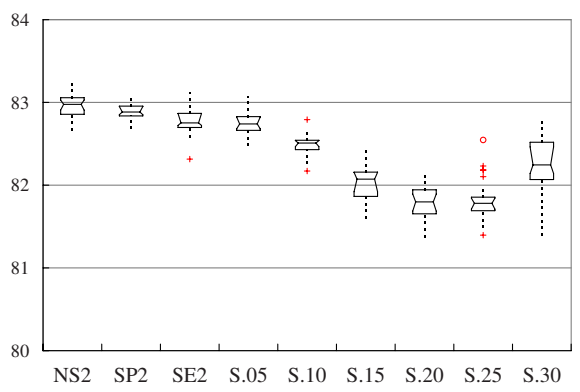

(e) ks4-500

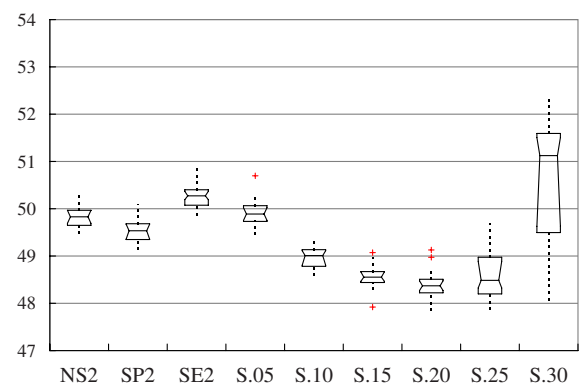

(b) ks2-1920

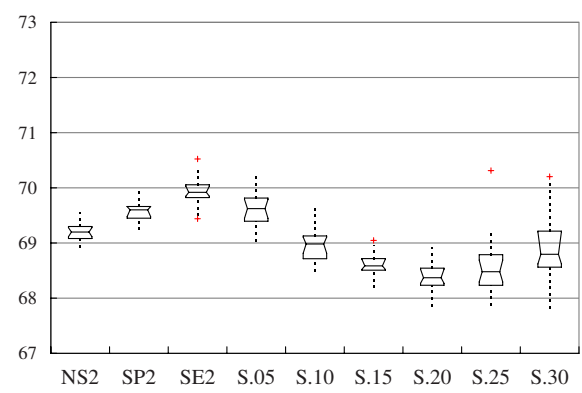

(d) ks3-1920

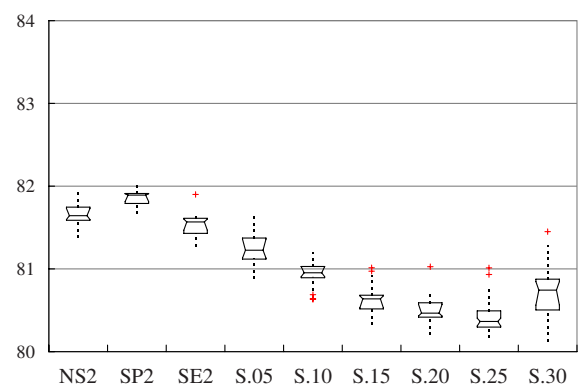

(f) ks4-1920

Fig. 1. Performance of algorithms on the multiple knapsack problem with respect to percentage of the non-covered objective space. NSGA2, SPEA2 and SEAMO2 are indicated by NS2, SP2 and SE2 respectively while S.xx indicates SEAMO2(RM) with a given value for $\sigma_{\text {mating. }}$. Results are given for 2 (graphs a-b), 3 (graphs c-d) and 4 (graphs e-f ) knapsacks with runs of 500 and 1920 generations.

on request) but we observed that increasing $\sigma_{\text {mating }}$ seems to have a negative impact on convergence and a positive impact on diversity. To illustrate this, we show in Figure 2 the offline non-dominated fronts after 30 independent runs of SEAMO2(RM) on the 2-knapsack problem. For better visualisation, we show the non-dominated fronts in a lower density (only solutions separated by a distance 


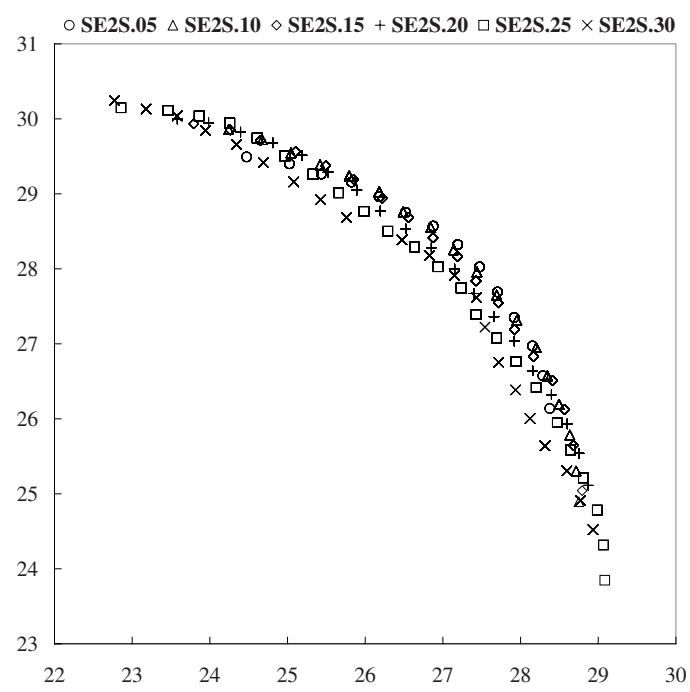

Fig. 2. Results of SEAMO2(RM) on the 2-knapsack and 750 items problem for six values of $\sigma_{\text {mating }}$. The horizontal axis represents profit in knapsack one and the vertical axis represents profit in knapsack two.

of at least 400 units in the objective space). We can see that higher $\sigma_{\text {mating }}$ values reduce the convergence of SEAMO2(RM) but increase diversity (this is similar to the observations by Ishibuchi and Shibata [9]). Therefore, in the next subsection we propose to adapt the mating pressure as the search progresses.

\subsection{Dynamic Setting of the Mating Pressure}

Now we describe how $\sigma_{\text {mating }}$ is adapted during the evolutionary search. This allows to improve both convergence and diversity of the population along with the evolutionary process. To dynamically change the value of $\sigma_{\text {mating }}$, we need first to establish the diff(range). As discussed in section 3.3. we select uniformly a value for $\sigma_{\text {mating }}$ in every generation within the 5 th and 95 th percentile of diff(range). This prevents the restricted mating becoming uniform selection (if $\sigma_{\text {mating }}$ is too low) or becoming a non-reproduction scheme (if $\sigma_{\text {mating }}$ is too high). Note that the mating pressure $\sigma_{\text {mating }}$ is set in an adaptive manner as diff(range) is adjusted after every generation to reflect the change of diversity (in the decision space) in the population. Then, the chosen value of $\sigma_{\text {mating }}$ will adjust as the population diversity changes. For example, in the first few generations, the population is less 'stable' with many randomly generated solutions provoking a high value of $\sigma_{\text {mating }}$. However, once the population is more 'stable', changes in the value of $\sigma_{\text {mating }}$ drive the population to evolve towards improving diversity (wider diff(range)) or improving convergence (smaller diff(range)).

As before, we carry out 30 independent runs of SEAMO2(RM) using the dynamic $\sigma_{\text {mating }}$. We also include the 'best results' obtained using Ishibuchi and 
Shibata's restricted mating strategy [10] and using the static mating strategy of section 3.3. These 'best results' are based on the average of the $\mathcal{S}$ metric over 30 independent runs. We used 90 combinations of values $\alpha=\{1,3,4, \ldots, 9,10\}$ and $\beta=\{1,2, \ldots, 9,10\}$ for Ishibuchi and Shibata's strategy and 11 different values of $\sigma_{\text {mating }}$ in the static mating strategy. These 'best results' are indicated as SE2I and SE2S in Figure 3 while SE2D indicates SEAMO2(RM) using the dynamic $\sigma_{\text {mating }}$. Figure 3 compares NSGA2, SPEA2, SEAMO2, SEAMO2 with Ishibuchi and Shibata's mating strategy, SEAMO2 with the static $\sigma_{\text {mating }}$ setting and SEAMO2 with the dynamic $\sigma_{\text {mating }}$ setting, with respect to the $\mathcal{S}$ metric. Table 1 shows the comparison with respect to the $\mathcal{C}$ metric.

For each knapsack problem, Figure 3 shows the average non-covered objective space (smaller values indicate better algorithm performance) at generations 500, 960 and 1920 side by side to facilitate comparison. It is clear that the dynamic setting of $\sigma_{\text {mating }}$ benefits SEAMO2 helping it to outperform NSGA2, SPEA2 and SEAMO2 as well as SEAMO2 with Ishibuchi and Shibata's mating strategy. Furthermore, both our static and dynamic mating strategies outperform Ishibuchi and Shibata's restricted mating strategy when incorporated into SEAMO2. In most cases, the dynamic strategy ourperforms the static one with the exception of the 2-knapsack problem with short and medium runs (graphs a-b in Figure 3). Table 1 shows the strong performance of SEAMO2D (the dynamic restricted mating incorporated in SEAMO2) particularly on problems with 3 and 4 knapsacks. From Figure 3 and Table 1 we can see that the dynamic mating strategy significantly improves diversity but it slightly worsens convergence in the higher dimension problem (4 knapsacks). We also notice an interesting result in that Ishibuchi and Shibata's strategy seems to worsen the performance of SEAMO2 (it was reported in [10] that Ishibuchi adn Shibata's strategy improves the performance of SPEA and NSGA2). This is more noticeable in the early stages of the evolutionary search (generations 500 and $960)$ in low dimension problems ( 2 and 3 knapsacks). We believe that Ishibuchi and Shibata's mating strategy conforms with the selection strategy in SPEA and NSGA2 where individuals are uniformly chosen using tournament selection. However, Ishibuchi and Shibata's mating strategy interferes with the selection strategy in SEAMO2 (Ishibuchi and Shibata's mating strategy chooses the first parent with binary tournaments while in SEAMO2 each individual acts as the first parent once). Figure 4 shows (in lower density as in Figure 2) the nondominated fronts over 30 independent runs on the 2-knapsack problem. We can see that SEAMO2(RM) using the dynamic mating strategy outperforms SPEA2 and NSGA2 but its convergence is just slightly lower than for SEAMO2. Overall, results in Figure 3 . Figure 4 and Table 1 give evidence that the dynamic setting of $\sigma_{\text {mating }}$ is beneficial for SEAMO2 on the three multiple knapsack problems.

In Figure 5 we compare the proposed assortative mating scheme using the static setting and using the dynamic setting over 30 independent runs for the 2 -knapsack problem with 750 items. The various static settings are indicated by SE2S.xx and the dynamic setting is indicated by SE2D. We can see that the $d y$ namic assortative mating scheme can simultaneously maintain the convergence 


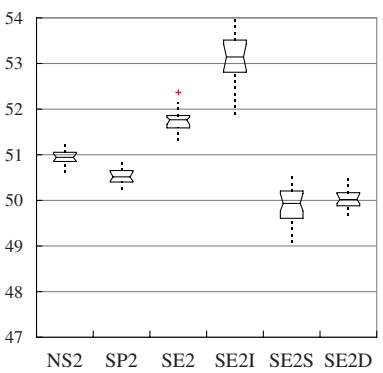

(a) ks2-500

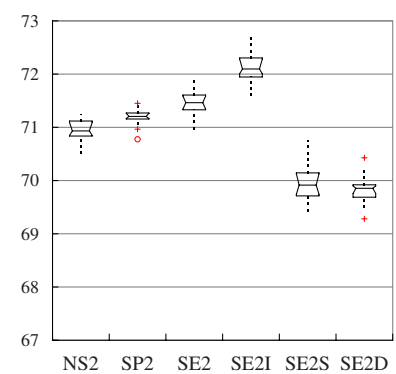

(d) ks3-500

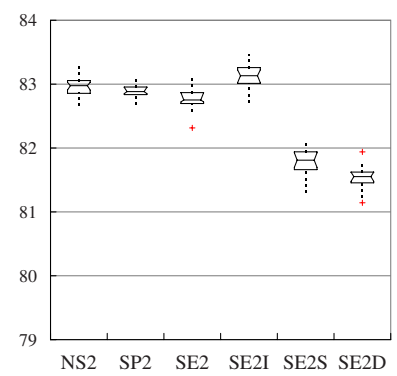

(g) ks4-500

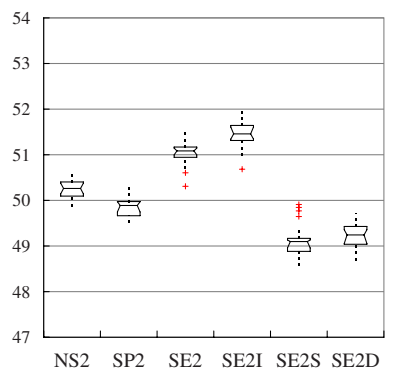

(b) ks2-960

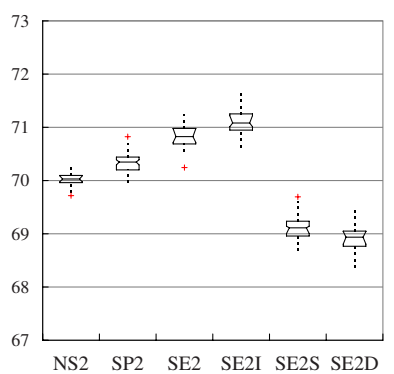

(e) ks3-960

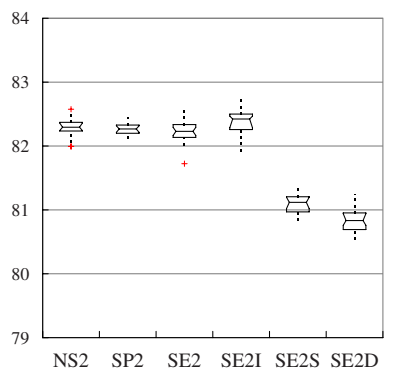

(h) ks4-960

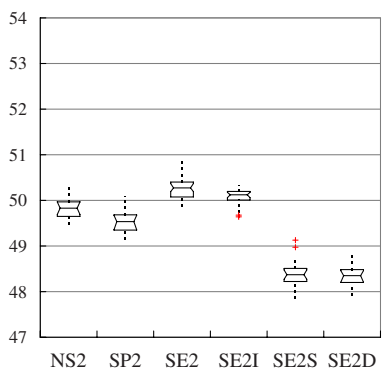

(c) ks2-1920

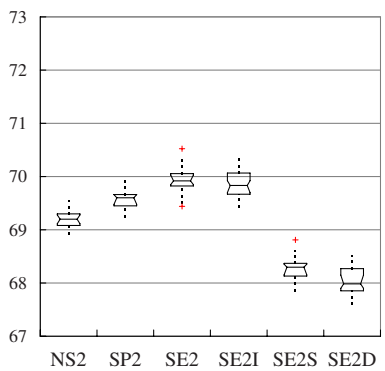

(f) ks3-750-1920

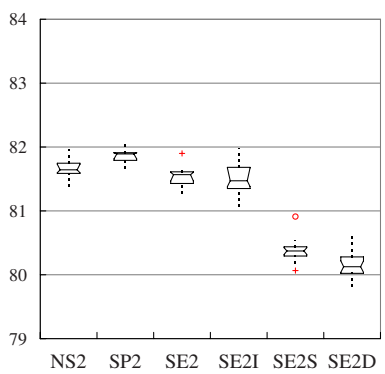

(i) ks4-1920

Fig. 3. Performance of algorithms on the multiple knapsack problem with respect to the percentage of the non-covered objective space. NSGA2, SPEA2 and SEAMO2 are indicated by NS2, SP2 and SE2 respectively. SE2I indicates SEAMO2 with Ishibuchi and Shibata's strategy, SE2S indicates SEAMO2 with the static setting and SE2D indicates SEAMO2 with the dynamic setting.

and the diversity of the population but the static setting can only give a positive effect on the convergence (using lower $\sigma_{\text {mating }}$ ) or on the diversity (using higher $\left.\sigma_{\text {mating }}\right)$ but not both at the same time. This shows that adapting the diff(range) (from where $\sigma_{\text {mating }}$ is chosen) according to the population diversity during evolution, helps to strike a balance between convergence and diversity. Of course, more elaborate methods for adapting the mating pressure can be investigated, but the proposed one points us on the right direction. 


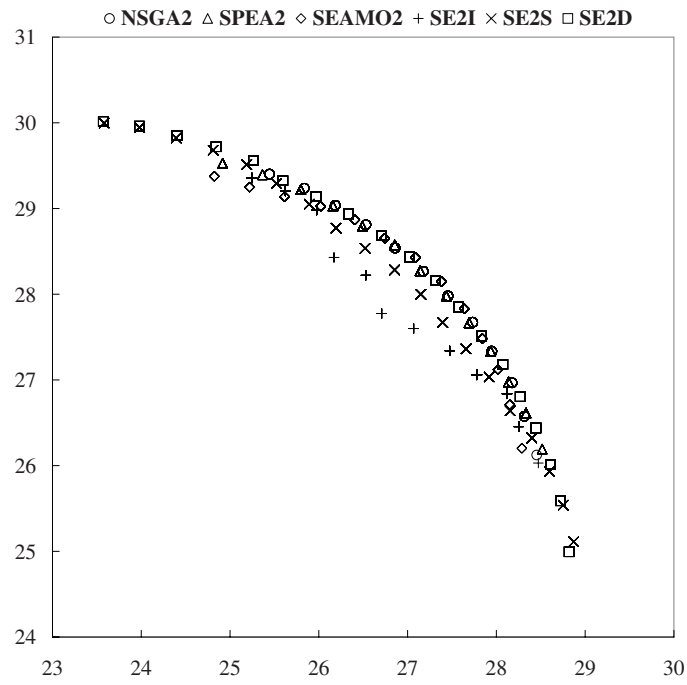

Fig. 4. Results comparing NSGA2, SPEA2, SEAMO2, SEAMO2 using Ishibuchi and Shibata's strategy (SE2I), SEAMO2 using the static mating strategy (SE2S) and SEAMO2(RM) using the dynamic mating strategy (SE2D) on the 2-knapsack problem with 750 items. The horizontal axis represents profit in knapsack one and the vertical axis represents profit in knapsack two.

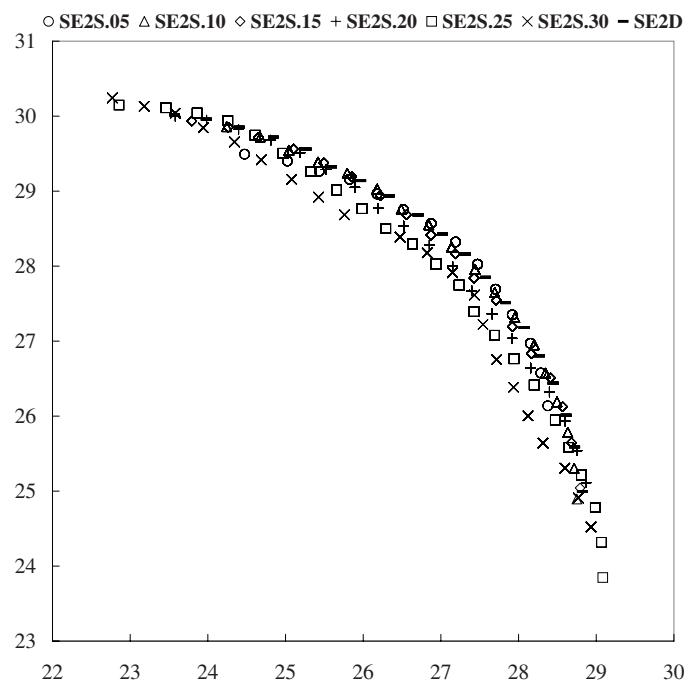

Fig. 5. Comparing the static and dynamic strategies for setting the mating pressure $\sigma_{\text {mating }}$ in SEAMO2(RM). These results are for the 2-knapsack problem with 750 items. The horizontal axis represents profit in knapsack one and the vertical axis represents profit in knapsack two. 
Table 1. Average values (standard deviation) of coverage of two sets $\mathcal{C}(\mathrm{A} \succeq \mathrm{B})$

\begin{tabular}{|l|l|c|c|c|c|c|c|c|c|c|}
\hline \multicolumn{10}{|c|}{$\mathcal{C}(\mathrm{A} \succeq \mathrm{B})$} \\
\hline \multicolumn{2}{|c|}{ Algorithm } & \multicolumn{2}{|c|}{2 knapsacks } & \multicolumn{2}{c|}{3 knapsacks } & \multicolumn{2}{c|}{4 knapsacks } \\
\hline A & B & 500 & 960 & 1920 & 500 & 960 & 1920 & 500 & 960 & 1920 \\
\hline NSGA2 & SEAMO2D & $3(7)$ & $12(16)$ & $24(20)$ & $0(0)$ & $0(0)$ & $0(0)$ & $0(0)$ & $0(0)$ & $0(0)$ \\
SPEA2 & & $2(3)$ & $8(7)$ & $18(16)$ & $0(0)$ & $0(0)$ & $0(0)$ & $0(0)$ & $0(0)$ & $0(0)$ \\
SEAMO2 & & $11(17)$ & $18(20)$ & $26(19)$ & $21(17)$ & $24(16)$ & $26(15)$ & $26(22)$ & $21(18)$ & $19(14)$ \\
SEAMO2I & & $0(0)$ & $0(0)$ & $0(1)$ & $0(0)$ & $0(0)$ & $2(5)$ & $0(0)$ & $0(1)$ & $1(3)$ \\
SEAMO2S & & $2(3)$ & $3(4)$ & $5(5)$ & $0(1)$ & $1(1)$ & $1(1)$ & $0(1)$ & $1(1)$ & $1(1)$ \\
\hline SEAMO2D & NSGA2 & $89(12)$ & $69(24)$ & $46(27)$ & $92(7)$ & $84(7)$ & $77(8)$ & $100(1)$ & $99(3)$ & $98(3)$ \\
& SPEA2 & $89(10)$ & $74(15)$ & $53(22)$ & $88(8)$ & $64(10)$ & $45(9)$ & $95(4)$ & $84(7)$ & $76(7)$ \\
& SEAMO2 & $76(34)$ & $60(38)$ & $47(34)$ & $34(29)$ & $24(25)$ & $18(19)$ & $16(20)$ & $15(18)$ & $12(12)$ \\
& SEAMO2I & $100(0)$ & $100(3)$ & $92(15)$ & $100(1)$ & $98(2)$ & $82(25)$ & $91(16)$ & $84(23)$ & $65(32)$ \\
& SEAMO2S & $80(8)$ & $82(11)$ & $83(10)$ & $86(6)$ & $83(6)$ & $78(8)$ & $79(6)$ & $75(7)$ & $67(7)$ \\
\hline
\end{tabular}

\section{Final Remarks}

This paper proposes a restricted mating scheme for evolutionary multi-objective (EMO) algorithms. This mating scheme is assortative because it selects parents based on their similarity in the decision space. Setting the mating pressure $\sigma_{\text {mating }}$ to a constant value provokes either convergence or diversity to be negatively affected. Therefore, the proposed scheme is adaptive because it varies $\sigma_{\text {mating }}$ taking into account the population diversity in the decision space. Our experiments show that the simple mechanism to adapt the mating pressure helps SEAMO2 (simple evolutionary algorithm for multi-objective optimisation) to improve its performance while striking a good balance between convergence and diversity. The proposed mating scheme can be incorporated into different EMO algorithms because it does not alter their original selection strategy. Future work contemplates comparison with other mating schemes proposed for EMO algorithms and on other problems such as nurse scheduling and job shop scheduling problems. We also intend to investigate other strategies to set the threshold $\sigma_{\text {mating }}$ to further improve diversity and convergence of EMO algorithms.

\section{References}

1. Blickle, T., Thiele, L.: A Comparison of Selection Schemes Used in Evolutionary Algorithms. Evolutionary Computation 4(4), 361-394 (1997)

2. Coello Coello, C.A., Van Veldhuizen, D.A., Lamont, G.B.: Evolutionary Algorithms for Solving Multi-objective Problems. Kluwer Academic Publishers, Dordrecht (2002)

3. De, S., Pal, S.K., Ghosh, A.: Genotypic and Phenotypic Assortative Mating in Genetic Algorithm. Information Sciences 105, 209-226 (1998)

4. Deb.: Kalyanmoy: Multi-objective Optimization Using Evolutionary Algorithms, Wiley, Chichester (2001) 
5. Deb, K., Pratap, A., Agarwal, S., Meyarivan, T.: A Fast and Elitist Multiobjective Genetic Algorithm: NSGA-II. IEEE Transactions on Evolutionary Computation 6(2), 182-197 (2002)

6. Goldberg, D.E.: Genetic Algorithms in Search, Optimisation and Machine Learning. Addison Wesley, Reading (1989)

7. Goldberg, D.E., Deb, K.: A Comparative Analysis of Selection Schemes Used in Genetic Algorithms. In: Rawlins, G.J.E. (ed.) Foundations of Genetic Algorithms, Morgan Kaufmann, San Francisco (1991)

8. Huang, C.F.: An Analysis of Mate Selection in Genetic Algorithms. Technical Report, Center for the Study of Complex Systems, University of Michgan (2001), (also as poster paper in the GECCO 2001 conference p. 766 (2001)

9. Ishibuchi, H., Shibata, Y.: An Empirical Study on the Effect of Mating Restriction on the Search Ability of EMO Algorithms. In: Fonseca, C.M., Fleming, P.J., Zitzler, E., Deb, K., Thiele, L. (eds.) EMO 2003. LNCS, vol. 2632, pp. 433-447. Springer, Heidelberg (2003)

10. Ishibuchi, H., Shibata, Y.: A Similarity-Based Mating Scheme for Evolutionary Multiobjective Optimization. In: The 2003 Genetic and Evolutionary Computation Conference, pp. 1065-1076 (2003)

11. Ishibuchi, H., Shibata, Y.: Mating Scheme for Controlling the Diversityconvergence Balance for Multi-objective Optimization. In: Deb, K., al., e. (eds.) GECCO 2004. LNCS, vol. 3102, pp. 1259-1271. Springer, Heidelberg (2004)

12. Ishibuchi, H., Shibata, Y.: Recombination of Similar Parents in EMO Algorithms. In: Coello Coello, C.A., Hernández Aguirre, A., Zitzler, E. (eds.) EMO 2005. LNCS, vol. 3410, pp. 265-279. Springer, Heidelberg (2005)

13. Kim, M., Hiroyasu, T., Miki, M., Watanabe, S.: SPEA2+: Improving The Performance of The Strength Pareto Evolutionary Algorithm 2. In: Yao, X., Burke, E.K., Lozano, J.A., Smith, J., Merelo-Guervós, J.J., Bullinaria, J.A., Rowe, J.E., Tiňo, P., Kabán, A., Schwefel, H.-P. (eds.) PPSN 2004. LNCS, vol. 3242, pp. 742-751. Springer, Heidelberg (2004)

14. Laumanns, M., Zitzler, E., Thiele, L.: On The Effects of Archiving, Elitism, and Density Based Selection in Evolutionary Multiobjective Optimization. In: Zitzler, E., Deb, K., Thiele, L., Coello Coello, C.A., Corne, D.W. (eds.) EMO 2001. LNCS, vol. 1993, pp. 181-196, Springer, Heidelberg (2001)

15. Mumford, C.L.: Simple Population Replacement Strategies for a Steady-State Multi-objective Evolutionary Algorithm. In: Deb, K., et al. (eds.) GECCO 2004. LNCS, vol. 3102, pp. 1389-1400. Springer, Heidelberg (2004)

16. Zitzler, E., Laumanns, M., Thiele, L.: SPEA2: Improving the Strength Pareto Evolutionary Algorithm for Multiobjective Optimization. In: Evolutionary Methods for Design, Optimisation and Control with Applications to Industrial Problems, pp. 95-100 (2002)

17. Zitzler, E., Thiele, L.: Multiobjective Evolutionary Algorithms: A Comparative Case Study and the Strength Pareto Approach. IEEE Transactions on Evolutionary Computation 3(4), 257-271 (1999) 